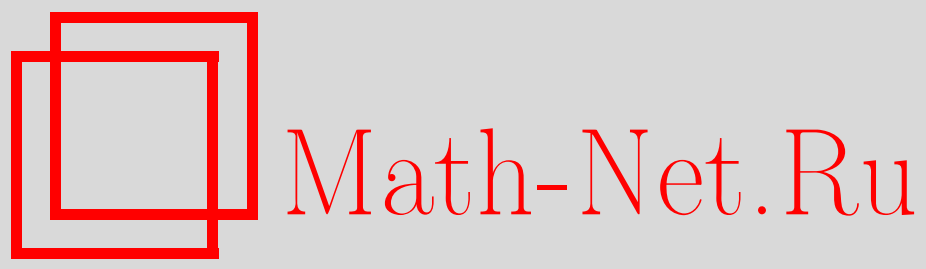

В. Г. Михайлов, Оценка точности сложной пуассоновской аппроксимации для распределения числа совпадающих цепочек, Теория вероятн. и ее примен., 2001, том 46, выпуск 4, 713-723

DOI: https://doi.org/10.4213/tvp3796

Использование Общероссийского математического портала MathNet.Ru подразумевает, что вы прочитали и согласны с пользовательским соглашением

http://www . mathnet.ru/rus/agreement

Параметры загрузки:

IP : 3.85 .7 .115

26 апреля 2023 г., 04:23:56

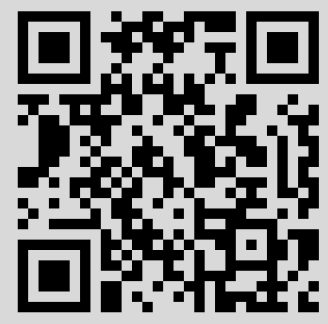




\title{
ОЩЕНКА ТОЧНОСТИ СЛОЖНОЙ ПУАССОНОВСКОЙ АППРОКСИМАЦИИ ДЛЯ РАСПРЕДЕЛЕНИЯ ЧИСЛА СОВПАДАЮЩИХ ЦЕПОЧЕК ${ }^{1)}$
}

\begin{abstract}
Пусть $X_{1}, \ldots, X_{m}$ и $Y_{1}, \ldots, Y_{n}$ - две последовательности независимых одинаково распределенных случайных величин, принимающих значения $1,2, \ldots$ С помощью специального варианта метода Стейна строится оценка точности аппроксимации распределения числа совпадений цепочек исходов $X_{i}, \ldots, X_{i+s-1}$ заданной длины $s$ в первой последовательности с цепочками исходов $Y_{j}, \ldots, Y_{j+s-1}$ во второй последовательности. В качестве аппроксимирующего выступает распределение суммы пуассоновского числа независимых случайных величин с геометрическим распределением.
\end{abstract}

Ключевые слова и фразы: длинные повторения, совпадения слов, оценки точности пуассоновской аппроксимации, сложное пуассоновское распределение, методы Стейна и Чена-Стейна.

Рассмотрим следующую задачу. Пусть имеются две последовательности $X_{1}, \ldots, X_{m}$ и $Y_{1}, \ldots, Y_{n}$ независимых одинаково распределенных случайных величин, принимающих значения $1,2, \ldots$. При этом считаем, что за случайной величиной $X_{m}$ следуют случайные величины $X_{1}, X_{2}, \ldots$, а за случайной величиной $Y_{n}$ следуют случайные величины $Y_{1}, Y_{2}, \ldots$. Циклическая структура, заданная таким образом на множестве индексов, слабо отражается на исследуемых свойствах последовательностей и использована главным образом для некоторого упрощения изложения.

Будем использовать обозначения

$$
\begin{aligned}
p_{k} & =\mathbf{P}\left\{X_{i}=k\right\}, & q_{k}=\mathbf{P}\left\{Y_{i}=k\right\}, \quad k=1,2, \ldots, \\
p & =\max _{k} p_{k} I\left\{q_{k}>0\right\}, & q=\max _{k} q_{k} I\left\{p_{k}>0\right\}
\end{aligned}
$$

*Математический институт им. В. А. Стеклова РАН, ул. Губкина, 8, 117966 Москва, ГСП-1, Россия.

1) Работа выполнена при поддержке Российского фонда фундаментальньх исследований (проект 96-01-00531) и Совета по грантам Президента РФ и государственной поддержки ведущих научньх школ (проект 96-15-96092). 
(здесь и далее $I\{\cdot\}$ обозначает индикатор случайного события или неслучайного множества),

$$
r=\max _{k} p_{k} q_{k}, \quad R=\sum_{k=1}^{\infty} p_{k} q_{k}
$$

Исследуется случайная величина

$$
\xi(m, n, s)=\sum_{i=1}^{m} \sum_{j=1}^{n} I\left\{\left(X_{i}, \ldots, X_{i+s-1}\right)=\left(Y_{j}, \ldots, Y_{j+s-1}\right)\right\}
$$

выражающая число совпадений цепочек исходов $X_{i}, \ldots, X_{i+s-1}$ заданной длины $s$ в первой последовательности с цепочками исходов $Y_{j}, \ldots, Y_{j+s-1}$ во второй последовательности. Величина $\xi(m, n, s)$ вместе с группой связанных с ней случайных величин и характеристик (длина наиболее длинного совпадения, число совпадающих участков и т.п.) играет существенную роль в математических моделях молекулярной биологии и генетики (см., например, библиографию в [1]).

Задача о совпадающих участках в паре последовательностей рассматривалась во многих статьях. Предельная теорема Пуассона для числа повторений участков длины не меньше заданной (в случае, когда элементы обеих последовательностей распределены одинаково) была доказана в работе [2], оценка скорости сходимости в этой теореме была получена в работе [3]. В последнем случае был использован метод ЧенаСтейна. Как следствия в этих работах были получены предельные теоремы и оценки в них для максимальной длины совпадающих участков. В литературе имеются также результаты о совпадениях участков в независимых реализациях последовательностей слабо зависимых случайных величин (см., например, [4], [5]). Довольно детально изучались вопросы, касающиеся длины максимального участка с заданной долей совпадений букв и максимального числа совпадений букв в словах заданной длины (см. [2], [6]-[8]). Здесь мы не касаемся этих вопросов.

Основной результат настоящей статьи формулируется следующим образом. Пусть $d(L(U), L(V))$ обозначает расстояние по вариации между распределениями случайных величин $U$ и $V$. Пусть $\lambda-$ среднее число пар повторившихся участков длины не меньше $s, \lambda=n m(1-R) R^{s}$, а $\mathrm{CP}(\Lambda)$, где $\Lambda=\left(\lambda_{1}, \lambda_{2}, \ldots\right)$, обозначает распределение случайной величины $\sum_{i=1}^{\infty} i \pi\left(\lambda_{i}\right)$, где $\pi\left(\lambda_{i}\right)$ - независимые случайные величины, распределенные по закону Пуассона с параметрами $\lambda_{i}$. Это обычное сложное пуассоновское распределение. Будем использовать обозначения $\Theta=\left(\theta_{1}, \theta_{2}, \ldots\right), \theta_{i}=\lambda(1-R) R^{i-1}$,

$$
d(\Theta)=d(L(\xi(m, n, s)), \mathrm{CP}(\Theta)), \quad S(R)=(1-R)(1-2 R) .
$$


Заметим, что распределение $\mathrm{CP}(\Theta)$ отвечает сумме пуассоновского (с параметром $\lambda$ ) числа независимых случайных слагаемых, имеющих геометрическое распределение (с параметром $R$ ).

Теорема 1. Пусть $2 \leqslant s<\min \{n, m\}, 0<R<1$. Тогда

$$
\begin{aligned}
d(\Theta) & =d(L(\xi(m, n, s)), \operatorname{CP}(\Theta)) \\
& <C(\lambda, R)\left(A \lambda+B \lambda^{2}\right)+\frac{4 \lambda^{2}}{n m R(1-R)}
\end{aligned}
$$

$2 \partial e$

$$
\begin{gathered}
C(\lambda, R) \leqslant \min \left\{1,(\lambda(1-R))^{-1}\right\} e^{\lambda} \\
A=\frac{2 s}{1-R}\left(2 s\left(\frac{r}{R}\right)^{s}+m p^{s}+n q^{s}\right), \quad B=\frac{2(4 s-3)}{(1-R)^{2}}\left(\frac{1}{n}+\frac{1}{m}\right) .
\end{gathered}
$$

Если при этом $0<R<\frac{1}{2}$, то можно взять

$$
C(\lambda, R)=\min \left\{1, \frac{1}{\lambda S(R)}\left[\frac{1}{4 \lambda S(R)}+(\ln (2 \lambda S(R)))_{+}\right]\right\} .
$$

Следствие 1. Пусть $n, m \rightarrow \infty u 2 \leqslant s<\min \{n, m\}$. Тогда, если $0<R \leqslant$ const $<1$ u $\lambda=O(1)$ uли если $0<R \leqslant$ const $<\frac{1}{2} u \lambda \rightarrow \infty$, mo

$$
d(\Theta)=O\left(\left(s\left(\frac{r}{R}\right)^{s}+m p^{s}+n q^{s}+\frac{\lambda}{n}+\frac{\lambda}{m}\right) s \ln \lambda\right) .
$$

Следствие 2. Пусть $n, m \rightarrow \infty$, a остальные параметры схемы меняются $\operatorname{ma\kappa ,~ито~} 2 \leqslant s<\min \{n, m\}, 0<R \leqslant$ const $<\frac{1}{2}, \lambda \rightarrow \infty u$ выполнено условие

$$
\left(s\left(\frac{r}{R}\right)^{s}+m p^{s}+n q^{s}+\frac{\lambda}{n}+\frac{\lambda}{m}\right) s \ln \lambda \longrightarrow 0 .
$$

Тогда распределение случайной величины

$$
\left(\xi(m, n, s)-\lambda(1-R)^{-1}\right)(1-R)(\lambda(1+R))^{-1 / 2}
$$

сходится $\kappa$ стандартному нормальному распределению.

3 а м е ч а н и е 1. Асимптотическое распределение случайной величины $\xi(m, n, s)$ представляет собой сложное пуассоновское распределение с производящей функцией $\mathbf{E} z^{\xi(m, n, s)}=\exp \{\lambda(z-1) /(1-z R)\}$ и имеет простой вероятностный смысл. В последовательностях $X_{1}, \ldots, X_{m}$ и $Y_{1}, \ldots, Y_{n}$ имеется асимптотически пуассоновское (с параметром $\lambda$ ) число $N(m, n, s)$ пар совпадающих между собой участков длины не 
меньше $s$. Длины этих участков асимптотически независимы и превосходят $s$ на случайные добавки с асимптотически геометрическим распределением (с параметром $R$ ) длины добавка.

В связи с этим приведем (без доказательства) оценку точности пуассоновской аппроксимации для распределения случайной величины $N(m, n, s)$. Обозначим через $\mathrm{Po}(\lambda)$ пуассоновское распределение с параметром $\lambda$.

Теорема 2. Пусть $R<1$ us $<\min \{n, m\}$. Тогда выполнено неравенство

$$
d(L(N(m, n, s)), \operatorname{Po}(\lambda))<(2 s+1)\left(1-e^{-\lambda}\right) B(m, n, s),
$$

2де

$$
B(m, n, s)=\frac{2 s}{1-R}\left(\frac{r}{R}\right)^{s}+m p^{s}+n q^{s}+(n+m)(1-R) R^{s} .
$$

Исследованию свойств случайной величины $N(m, n, s)$ будет посвящена отдельная статья.

3 а м е ч а н и е 2. В случае, когда все величины $X_{1}, \ldots, X_{m}$ и $Y_{1}, \ldots, Y_{n}$ имеют одинаковые распределения, оценка точности пуассоновской аппроксимации для распределения случайной величины $N(m, n, s)$ была получена в работе [3].

Поскольку в теореме 1 использовано ограничение $s \geqslant 2$, рассмотрим отдельно случай $s=1$. Он заметно проше, и это позволяет нам несколько расширить постановку, допустив, в частности, неодинаковые распределения у случайных величин в каждой из последовательностей $X_{1}, \ldots, X_{m}$ и $Y_{1}, \ldots, Y_{n}$.

Введем обозначения

$$
\begin{array}{ll}
P_{k}=\sum_{i=1}^{m} \mathbf{P}\left\{X_{i}=k\right\}, & Q_{k}=\sum_{j=1}^{n} \mathbf{P}\left\{Y_{j}=k\right\}, \\
p_{k}=\max _{i} \mathbf{P}\left\{X_{i}=k\right\}, & q_{k}=\max _{j} \mathbf{P}\left\{Y_{j}=k\right\} .
\end{array}
$$

Пусть задано некоторо множество $A \subseteq\{1,2, \ldots\}$ и $\lambda(A)=\sum_{k \in A} P_{k} Q_{k}$.

Определим случайную величину

$$
\xi(m, n ; A)=\sum_{i=1}^{m} \sum_{j=1}^{n} I\left\{X_{i}=Y_{j} \in A\right\} .
$$

Очевидно, что $\xi(m, n ;\{1,2, \ldots\})=\xi(m, n, 1)$ и $\mathbf{E} \xi(m, n ; A)=\lambda(A)$.

Теорема 3. Выполняется неравенство

$$
\begin{aligned}
& d(L(\xi(m, n ; A)), \operatorname{Po}(\lambda(A))) \\
& \quad<\frac{1-e^{-\lambda(A)}}{\lambda(A)}\left(\sum_{k \in A} P_{k} Q_{k}\left(\left(1+q_{k}\right) P_{k}+\left(1+p_{k}\right) Q_{k}\right)\right) .
\end{aligned}
$$


3 а м е ч а н и е 3 . Из теоремы 3 вытекает, что достаточными для сближения распределения величины $\xi(m, n ; A)$ с законом Пуассона с параметром $\lambda$ являются условия

$$
\lambda(A) \rightarrow \lambda, \quad \sum_{k \in A} P_{k} Q_{k}\left(\left(1+q_{k}\right) P_{k}+\left(1+p_{k}\right) Q_{k}\right) \longrightarrow 0 .
$$

Нетрудно убедиться также, что

$$
\left\{\sum_{k \in A} P_{k} \rightarrow 0\right\} \text { или }\left\{\sum_{k \in A} Q_{k} \rightarrow 0\right\} \Longrightarrow\{\mathbf{P}\{\xi(m, n ; A)=0\} \longrightarrow 1\} .
$$

Следующая теорема показывает, что в случае равновероятных распределений указанные в (9) и (10) достаточные условия являются также и необходимыми.

Пусть распределения случайных величин $X_{i}$ и $Y_{j}$ равномерны:

$$
\begin{aligned}
& \mathbf{P}\left\{X_{i}=k\right\}=M^{-1}, \quad i=1, \ldots, m, k=1, \ldots, M, \\
& \mathbf{P}\left\{Y_{j}=k\right\}=N^{-1}, \quad j=1, \ldots, n, k=1, \ldots, N,
\end{aligned}
$$

и $A=\{1, \ldots, K\}, K \leqslant M, N$. Тогда

$$
P_{k}=m M^{-1}, \quad Q_{k}=n N^{-1}, \quad \lambda(A)=K m M^{-1} n N^{-1} .
$$

Теорема 4. Пусть в данной схеме $m, n \rightarrow \infty u \lambda(A) \rightarrow \lambda$, аде $0<$ $\lambda<\infty$.

а) Пусть выполнено условие $K \mathrm{mM}^{-1} \rightarrow 0$ или $K n N^{-1} \rightarrow 0$. Тогда

$$
\mathbf{P}\{\xi(m, n ; A)=0\} \longrightarrow 1 .
$$

б) Пусть выполнено условие $m M^{-1}+n N^{-1} \rightarrow 0$. Тогда

$$
L(\xi(m, n ; A)) \rightarrow \operatorname{Po}(\lambda)
$$

в) Пусть при некотором $\mu, 0<\mu<\infty$, выполнено соотношение $\min \left\{\left|K m M^{-1}-\mu\right|,\left|K n N^{-1}-\mu\right|\right\} \longrightarrow 0 u K \rightarrow \infty$. Тогда предельным для случайной величины $\xi(m, n ; A)$ является сложное пуассоновское распределение с производящей функиией

$$
\exp \left\{\mu\left(\exp \left\{\lambda \mu^{-1}(z-1)\right\}-1\right)\right\}
$$

г) Пусть $K=$ const, $m M^{-1} \rightarrow \mu, n N^{-1} \rightarrow \nu, 0<\mu, \nu<\infty$, $\lambda=K \mu \nu$. Тогда предельным для случайной величины $\xi(m, n ; A)$ является распределение выражения $\sum_{k=1}^{K} \pi_{k}(\mu) \pi_{k}(\nu)$, составленного из независимых случайных величин $\pi_{1}(\mu), \ldots, \pi_{K}(\mu), \pi_{1}(\nu), \ldots, \pi_{K}(\nu)$, pacnределенных по закону Пуассона с указанным в скобках параметром. 
3 а м е ч а н и е 4. Условие п. а) теоремы 4 эквивалентно соотношению $m M^{-1}+n N^{-1} \rightarrow \infty$. В п. б) изучена ситуация, когда эта сумма стремится к нулю. Условия пункта в) включают в себя два частных случая: $m M^{-1} \rightarrow 0, n N^{-1} \rightarrow \lambda \mu^{-1}$ и $m M^{-1} \rightarrow \lambda \mu^{-1}, n N^{-1} \rightarrow 0$ и всевозможные комбинации этих условий на подпоследовательностях. Случаю, когда $m M^{-1} \rightarrow \mu>0$ и $n N^{-1} \rightarrow \nu>0$, отвечает п. г) теоремы. Таким образом, в теореме 4 приведен полный спектр предельных распределений для величины $\xi(m, n ; A)$ в рассмотренном случае. Из этого факта заключаем, что в данном случае (см. (11))

$$
\{\mathbf{P}\{\xi(m, n ; A)=0\} \longrightarrow 1\} \Longrightarrow\left\{\sum_{k \in A} P_{k} \rightarrow 0\right\} \text { или }\left\{\sum_{k \in A} Q_{k} \rightarrow 0\right\},
$$

а из условия $\{\lambda(A) \rightarrow \lambda \& L(\xi(m, n ; A)) \rightarrow \operatorname{Po}(\lambda)\}$ следует второе из условий (9).

Перейдем к доказательствам. Нам понадобится следующая теорема о суммах случайных индикаторов (см. [9] или [10]).

Пусть $\Gamma$ - произвольный конечный набор индексов; $I_{a}, a \in \Gamma$, случайные индикаторы, $W=\sum_{a \in \Gamma} I_{a}$. Для каждого $I_{a}$ разделим некоторым образом множество $Г$ на четыре непересекающихся множества: $\{a\}, \Gamma_{a}^{\nu s}, \Gamma_{a}^{b}, \Gamma_{a}^{\nu w}$, и положим

$$
U_{a}=\sum_{b \in \Gamma_{a}^{\nu s}} I_{b}, \quad V_{a}=\sum_{b \in \Gamma_{a}^{b}} I_{b} .
$$

Определим набор $\Lambda=\left(\lambda_{1}, \ldots, \lambda_{D+1}, 0, \ldots\right)$, где

$$
\lambda_{i}=i^{-1} \sum_{a \in \Gamma} \mathbf{E}\left\{I_{a} I\left\{I_{a}+U_{a}=i\right\}\right\}, \quad D=\max _{a}\left|\Gamma_{a}^{\nu s}\right| .
$$

Введем обозначение $\varphi=\sum_{a \in \Gamma} \sum_{i=1}^{\left|\Gamma_{a}^{\nu s}\right|+1} \varphi_{a i}$, где

$$
\varphi_{a i}=\mathbf{E}\left|\mathbf{E}\left\{I_{a} I\left\{I_{a}+U_{a}=i\right\} \mid\left(I_{b}: b \in \Gamma_{a}^{\nu w}\right)\right\}-\mathbf{E}\left\{I_{a} I\left\{I_{a}+U_{a}=i\right\}\right\}\right| .
$$

Теорема 5. При любом выборе указанных выше множеств

$$
\begin{aligned}
& d(L(W), \operatorname{CP}(\Lambda)) \\
& \quad \leqslant c_{1}(\Lambda) \varphi+c_{2}(\Lambda) \sum_{a \in \Gamma}\left(\left(\mathbf{E} I_{a}\right)^{2}+\mathbf{E} I_{a} \mathbf{E}\left(U_{a}+V_{a}\right)+\mathbf{E} I_{a} V_{a}\right),
\end{aligned}
$$

аде

$E \operatorname{csu} \nu \lambda_{\nu} \downarrow 0$ npu $\nu \uparrow \infty, m o$

$$
\max \left\{c_{1}(\Lambda), c_{2}(\Lambda)\right\} \leqslant \min \left\{1, \frac{1}{\lambda_{1}}\right\} \exp \left\{\sum_{s=1}^{\infty} \lambda_{s}\right\} .
$$

$$
\begin{aligned}
& c_{1}(\Lambda)= \begin{cases}1, & \lambda_{1}-2 \lambda_{2} \leqslant 1, \\
\left(\lambda_{1}-2 \lambda_{2}\right)^{-1 / 2}\left[2-\left(\lambda_{1}-2 \lambda_{2}\right)^{-1 / 2}\right], & \lambda_{1}-2 \lambda_{2}>1,\end{cases} \\
& c_{2}(\Lambda)=\min \left\{1,\left(\lambda_{1}-2 \lambda_{2}\right)^{-1}\left[\left(4\left(\lambda_{1}-2 \lambda_{2}\right)\right)^{-1}+\left(\ln 2\left(\lambda_{1}-2 \lambda_{2}\right)\right)_{+}\right]\right\} .
\end{aligned}
$$


Оценка (13) доказана в работе [9] с помощью результатов работы [11], неравенства и выражения для коэффициентов получены в [11] (подробности можно найти в обзорной статье [10]).

Д о к а з а т е ль с т в о т е о ре м ы 1 . Воспользуемся результатом теоремы 5 . В нашем случае $\Gamma=\{a=(i, j): 1 \leqslant i \leqslant n, 1 \leqslant j \leqslant m\}$, $I_{a}=I\left\{\left(X_{i}, \ldots, X_{i+s-1}\right)=\left(Y_{j}, \ldots, Y_{j+s-1}\right)\right\}$. Возьмем

$$
\begin{aligned}
\Gamma_{a}^{\nu s} & =\left\{b=\left(i^{\prime}, j^{\prime}\right) \in \Gamma \backslash\{a=(i, j)\}:-s<i^{\prime}-i=j^{\prime}-j<s\right\} \\
\Gamma_{a}^{\nu w} & =\left\{b=\left(i^{\prime}, j^{\prime}\right) \in \Gamma: \min \left\{\left|i^{\prime}-i\right|,\left|j^{\prime}-j\right|\right\}>2 s-1\right\}, \\
\Gamma_{a}^{b} & =\Gamma \backslash\left(\{a\} \cup \Gamma_{a}^{\nu s} \cup \Gamma_{a}^{\nu w}\right) .
\end{aligned}
$$

В силу этих определений $D+1=2 s-1$, т.е. в описании сопровождающего сложного пуассоновского распределения участвуют $2 s-1$ величин $\lambda_{\nu}$, а именно $\lambda_{1}, \ldots, \lambda_{2 s-1}$. Событие $\left\{I_{a}=1, I_{a}+U_{a}=\nu\right\}$ при $\nu=1, \ldots, s-1$ является объединением $\nu$ несовместных событий

$$
\left\{I_{a-k} \neq 1, I_{a-k+1}=\cdots=I_{a-k+\nu}=1, I_{a-k+\nu+1} \neq 1\right\},
$$

где $k=1, \ldots, \nu$ и считается, что $a-k=(i-k, j-k)$. Вероятность каждого из этих событий равна $(1-R)^{2} R^{s+\nu-1}$. Поэтому при $\nu=1, \ldots, s-1$

$$
\begin{aligned}
\lambda_{\nu} & =\frac{1}{\nu} \sum_{a \in \Gamma} \mathbf{E}\left\{I_{a} I\left\{I_{a}+U_{a}=\nu\right\}\right\} \\
& =n m(1-R)^{2} R^{s+\nu-1}=\lambda(1-R) R^{\nu-1},
\end{aligned}
$$

где $\lambda=n m(1-R) R^{s}$ - среднее число отрезков повторений длины не меньше $s$.

При $\nu=s, \ldots, 2 s-2$ событие $\left\{I_{a}=1, I_{a}+U_{a}=\nu\right\}$ является объединением $2 s-\nu-2$ несовместных событий

$$
\left\{I_{a-k} \neq 1, I_{a-k+1}=\cdots=I_{a-k+\nu}=1, I_{a-k+\nu+1} \neq 1\right\},
$$

где $k=\nu-s+1, \ldots, s-2$, и двух событий

$$
\begin{aligned}
& \left\{I_{a-s+1}=\cdots=I_{a-s+\nu}=1, I_{a-s+\nu+1} \neq 1\right\}, \\
& \left\{I_{a+s-\nu-1} \neq 1, I_{a+s-\nu}=\cdots=I_{a+s-1}=1\right\} .
\end{aligned}
$$

Вероятность каждого из этих двух событий равна $(1-R) R^{s+\nu-1}$. Поэтому при $\nu=s, \ldots, 2 s-2$

$$
\lambda_{\nu}=\nu^{-1} \lambda(1-R) R^{\nu-1}(2 s-\nu+2 R) .
$$

При $\nu=2 s-1$ выполняется равенство $\left\{I_{a}=1, I_{a}+U_{a}=\nu\right\}=\left\{I_{a-s+1}=\right.$ $\left.\cdots=I_{a+s-1}=1\right\}$. Вероятность этого события равна $R^{3 s-2}$. Поэтому

$$
\lambda_{2 s-1}=\frac{\lambda R^{2 s-2}}{(2 s-1)(1-R)} .
$$


Выведем теперь оценки для слагаемых в правой части (13). Очевидно, в нашем случае

$$
\varphi=0, \quad \mathbf{E} I_{a}=R^{s}
$$

$\mathbf{E} I_{a}\left(\mathbf{E} I_{a}+\mathbf{E} U_{a}+\mathbf{E} V_{a}\right)=(n m-(n-4 s+3)(m-4 s+3)) R^{2 s}$.

Самое главное теперь - вычислить или оценить $\mathbf{E} I_{a} V_{a}$. Выделим в $\Gamma_{a}^{b}$ подмножества

$$
\begin{aligned}
\Gamma_{a}^{*} & =\left\{b=\left(i^{\prime}, j^{\prime}\right) \in \Gamma_{a}^{b}: \min \left\{\left|i^{\prime}-i\right|,\left|j^{\prime}-j\right|\right\}<s\right\} \backslash \Gamma_{a}^{\nu s}, \\
\Gamma_{a}^{* *} & =\left\{b=\left(i^{\prime}, j^{\prime}\right) \in \Gamma_{a}^{*}: \max \left\{\left|i^{\prime}-i\right|,\left|j^{\prime}-j\right|\right\}<s\right\} .
\end{aligned}
$$

При $\left(i^{\prime}, j^{\prime}\right) \in \Gamma_{a}^{* *}$, как нетрудно проверить, следуя рассуждениям доказательства леммы 2 из [12], $\mathbf{E} I_{a} I_{b} \leqslant r^{s}$ (напомним, что $r=\max _{k} p_{k} q_{k}$ ). При $\left(i^{\prime}, j^{\prime}\right) \in \Gamma_{a}^{*} \backslash \Gamma_{a}^{* *}$, если $\left|i^{\prime}-i\right|<s$, то выполняется неравенство $\mathbf{E} I_{a} I_{b} \leqslant \mathbf{E} I_{a} q^{s}$, а если $\left|j^{\prime}-j\right|<s$, то выполняется неравенство $\mathbf{E} I_{a} I_{b} \leqslant$ $\mathbf{E} I_{a} p^{s}$. Поэтому $\sum_{b \in \Gamma_{a}^{*}} \mathbf{E} I_{a} I_{b}<4 s^{2} r^{2}+\mathbf{E} I_{a}\left(2 s m p^{s}+2 s n q^{s}\right)$. Кроме того, $\mathbf{E} I_{a} I_{b}=\mathbf{E} I_{a} \mathbf{E} I_{b}$ при $b \in \Gamma_{a}^{b} \backslash \Gamma_{a}^{*}$. Поэтому

$$
\begin{aligned}
\mathbf{E} I_{a} V_{a}< & \sum_{b \in \Gamma_{a}^{*}} \mathbf{E} I_{a} I_{b}+\mathbf{E} I_{a} \mathbf{E} V_{a}<4 s^{2} r^{2}+2 s R^{2}\left(m p^{s}+n q^{s}\right) \\
& +(n m-(n-4 s+3)(m-4 s+3)) R^{2 s} .
\end{aligned}
$$

Подставив (17) и (18) в оценку теоремы 5, получим после некоторых упрощений неравенство

$$
d(L(\xi(m, n, s)), \operatorname{CP}(\Lambda))<c_{2}(\Lambda)\left(A \lambda+B \lambda^{2}\right) .
$$

Теперь о множителе $c_{2}(\Lambda)$. Оценка (4) следует непосредственно из теоремы 5 и наших определений. Воспользуемся условием $R<\frac{1}{2}$. В силу (14) $\lambda_{1}-2 \lambda_{2}=\lambda S(R)=\lambda(1-R)(1-2 R)$. Поэтому в этом случае

$$
c_{2}(\Lambda) \leqslant C(\lambda, R)
$$

с указанным в (5) значением $C(\lambda, R)$.

Оценим теперь расстояние между сложными пуассоновскими распределениями $\mathrm{CP}(\Lambda)$ с $\Lambda=\left(\lambda_{1}, \ldots, \lambda_{2 s-1}, 0, \ldots\right)$ и $\mathrm{CP}(\Theta)$ с $\Theta=\left(\theta_{1}, \theta_{2}, \ldots\right)$, $\theta_{i}=\lambda(1-R) R^{i-1}$. Последнее, как уже отмечалось, отвечает сумме пуассоновского числа (с параметром $\lambda$ ) независимых слагаемых, имеющих геометрическое распределение с параметром $R$. Воспользуемся тем, что

$$
d(\operatorname{CP}(\Lambda), \operatorname{CP}(\Theta)) \leqslant \sum_{i=1}^{2 s-1}\left|\lambda_{i}-\theta_{i}\right|+\sum_{i=2 s}^{\infty} \theta_{i} .
$$


Поэтому (используем (14)-(16), а также неравенства $s \geqslant 2$ и $R<\frac{1}{2}$ )

$$
\begin{aligned}
d(\mathrm{CP}(\Lambda), \mathrm{CP}(\Theta)) & \leqslant\left|\lambda_{s}-\theta_{s}\right|+\sum_{i=s+1}^{2 s-1}\left|\lambda_{i}-\theta_{i}\right|+\sum_{i=2 s}^{\infty} \theta_{i} \\
& \leqslant \frac{2 R}{s} \lambda(1-R) R^{s-1}+\lambda R^{s}+\lambda \frac{R^{2 s-1}}{1-R} \\
& \leqslant 4 \lambda R^{s}=\frac{4 \lambda^{2}}{n m(1-R)} .
\end{aligned}
$$

Используя (19), (21) и неравенство треугольника, получаем оценку (3). Теорема 1 доказана.

Утверждение следствия 1 вытекает непосредственно из оценки (3).

3 а м е ч а н и е 5 . Дополнительное слагаемое $d(\mathrm{CP}(\Lambda), \mathrm{CP}(\Theta))$, возникающее в оценке (3) из-за перехода к аппроксимации исследуемого распределения посредством распределения суммы пуассоновского числа геометрически распределенных слагаемых, не вносит сушественного вклада в (5) и оценку (6) следствия 1.

Доказательство следствия 2 проводится по схеме доказательства следствия из теоремы 3 в работе [13]. Согласно утверждению следствия 1 , в данном случае распределение случайной величины $\xi(m, n, s)$ неограниченно сближается со сложным пуассоновским распределением c производящей функцией $\exp \{\lambda(z-1) /(1-z R)\}$. Последнее в свою очередь асимптотически нормально с параметрами $\lambda(1-R)^{-1}$ (среднее) и $\lambda(1+R)(1-R)^{-2}$ (дисперсия). Используя эти свойства, получаем утверждение следствия 2.

Д ок а з а т е л ь с т в о т е о ре м ы 3 . Воспользуемся следующей ощенкой метода Чена-Стейна (см., например, [14]).

Пусть $\Gamma$ - произвольный конечный набор индексов, $\tilde{I}_{a}, a \in \Gamma$, случайные индикаторы, $\widetilde{W}=\sum_{a \in \Gamma} \tilde{I}_{a}$. Для каждого $\tilde{I}_{a}$ разделим некоторым образом множество $\Gamma$ на три непересекающихся множества: $\{a\}$, $\Gamma_{a}^{s}, \Gamma_{a}^{w}$, и положим

$$
\tilde{U}_{a}=\sum_{b \in \Gamma_{a}^{s}} \tilde{I}_{b} \quad \text { и } \quad \tilde{\varphi}_{a}=\mathbf{E}\left|\mathbf{E}\left\{\tilde{I}_{a} \mid\left(\tilde{I}_{b}: b \in \Gamma_{a}^{w}\right)\right\}-\mathbf{E} \tilde{I}_{a}\right| .
$$

Теорема 6 ([14]). Пусть $\widetilde{\lambda}=\mathbf{E} \widetilde{W}$. При любом выборе указанных выие множеств

$$
\begin{aligned}
d(L(\widetilde{W}), \operatorname{Po}(\widetilde{\lambda})) \leqslant & \min \left\{1, \frac{1}{\sqrt{\tilde{\lambda}}}\right\} \sum_{a} \widetilde{\varphi}_{a} \\
& +\frac{1-e^{-\widetilde{\lambda}}}{\tilde{\lambda}}\left(\sum_{a} \mathbf{E} \tilde{I}_{a}\left(\mathbf{E} \tilde{I}_{a}+\mathbf{E} \widetilde{U}_{a}\right)+\sum_{a} \mathbf{E} \tilde{I}_{a} \widetilde{U}_{a}\right) .
\end{aligned}
$$


В нашем случае $\tilde{\lambda}=\lambda(A), \tilde{I}_{a}=I\left\{X_{i}=Y_{j} \in A\right\}$,

$$
\Gamma=\{a=(i, j): 1 \leqslant i \leqslant n, 1 \leqslant j \leqslant m\} .
$$

Возьмем

$$
\begin{aligned}
\Gamma_{a}^{s} & =\left\{b=\left(i^{\prime}, j^{\prime}\right) \in \Gamma: i^{\prime} \neq i, j^{\prime}=j \quad \text { или } \quad i^{\prime}=i, j^{\prime} \neq j\right\} \\
\Gamma_{a}^{w} & =\left\{b=\left(i^{\prime}, j^{\prime}\right) \in \Gamma: i^{\prime} \neq i, j^{\prime} \neq j\right\} .
\end{aligned}
$$

Выведем оценки для слагаемых в правой части (22) в нашем случае. Очевидно, что в нашем случае

$$
\begin{aligned}
& \sum_{a} \widetilde{\varphi}_{a}=0, \quad \mathbf{E} \tilde{I}_{a}=\sum_{k \in A} \mathbf{P}\left\{X_{i}=k\right\} \mathbf{P}\left\{Y_{j}=k\right\} \\
& \mathbf{E} \tilde{I}_{a}\left(\mathbf{E} \tilde{I}_{a}+\mathbf{E} \tilde{U}_{a}\right) \\
& \leqslant \sum_{k \in A}\left\{\mathbf{P}\left\{X_{i}=k\right\} \mathbf{P}\left\{Y_{j}=k\right\}\right. \\
& \left.\quad \times\left(\sum_{i^{\prime}=1}^{m} \mathbf{P}\left\{X_{i^{\prime}}=k\right\} \mathbf{P}\left\{Y_{j}=k\right\}+\sum_{j^{\prime}=1}^{n} \mathbf{P}\left\{X_{i}=k\right\} \mathbf{P}\left\{Y_{j^{\prime}}=k\right\}\right)\right\} .
\end{aligned}
$$

Поэтому

$$
\sum_{a} \mathbf{E} \tilde{I}_{a}\left(\mathbf{E} \tilde{I}_{a}+\mathbf{E} \tilde{U}_{a}\right) \leqslant \sum_{k \in A} P_{k} Q_{k}\left(q_{k} P_{k}+p_{k} Q_{k}\right) .
$$

Аналогично

$$
\begin{aligned}
\mathbf{E} \tilde{I}_{a} \widetilde{U}_{a} & =\sum_{k \in A}\left(\sum_{\substack{i^{\prime}=1 \\
i^{\prime} \neq i}}^{m} \mathbf{P}\left\{X_{i}=X_{i^{\prime}}=Y_{j}=k\right\}+\sum_{\substack{j^{\prime}=1 \\
j^{\prime} \neq j}}^{n} \mathbf{P}\left\{X_{i}=Y_{j}=Y_{j^{\prime}}=k\right\}\right) \\
& \leqslant \sum_{k \in A} \mathbf{P}\left\{X_{i}=k\right\} \mathbf{P}\left\{Y_{j}=k\right\}\left(\sum_{i^{\prime}=1}^{m} \mathbf{P}\left\{X_{i^{\prime}}=k\right\}+\sum_{j^{\prime}=1}^{n} \mathbf{P}\left\{Y_{j^{\prime}}=k\right\}\right) .
\end{aligned}
$$

Поэтому

$$
\sum_{a \in \Gamma} \mathbf{E} \tilde{I}_{a} \widetilde{U}_{a} \leqslant \sum_{k \in A} P_{k} Q_{k}\left(P_{k}+Q_{k}\right) .
$$

Из наших определений и оценок (22)-(25) следует (8). Теорема 3 доказана.

Доказа те льс т во те оремы 4. По условиям п.а) теоремы в одной из последовательностей среднее число встретившихся в ней знаков из множества $A$, т.е. тех знаков, для которых может быть зафиксировано совпадение, стремится к нулю. Значит, к нулю стремятся вероятность наличия хотя бы одного такого знака и вероятность фиксации совпадения знаков.

Утверждение п. б) является прямым следствием теоремы 3. 
При доказательстве п. в) ограничимся случаем $K m M^{-1} \rightarrow \mu$. Заметим, что при этом условии число появившихся в первой последовательности знаков из множества $A$ имеет в пределе распределение Пуассона с параметром $\mu$. С вероятностью, стремящейся к единице, все эти элементы различны. В тех же условиях для любого фиксированного набора элементов из алфавита второй последовательности их абсолютные частоты асимптотически независимы и имеют в пределе распределение Пуассона с параметром $\lambda \mu^{-1}=\lim n N^{-1}$. Используя эти свойства, получаем сходимость к указанному распределению. Аналогично рассматриваются остальные случаи, описанные в условиях п. в). Подобным образом доказываются и п. г). Теорема 4 доказана.

Автор признателен А. М. Зубкову за ряд полезных замечаний.

\section{СПИСОК ЛИТЕРАТУРЫ}

1. Waterman M., Vingron $M$. Sequence comparison significance and Poisson approximation. - Statist. Sci., 1994, v. 9, № 3, p. 367-381.

2. Arratia R., Gordon L., Waterman M.S. An extreme value theory for sequence matching. - Ann. Statist., 1986, v. 14, № 3, p. 971-993.

3. Новак С. Ю. Пуассонова аппроксимация числа длинных «повторов» в случайных последовательностях. - Теория вероятн. и ее примен., 1994, т. 39, в. 4, с. 731-742.

4. Karlin S., Ost F. Counts of long aligned word matches among random letter sequences. - Adv. Appl. Probab., 1987, v. 19, № 3, p. 293-351.

5. Karlin S., Ost F. Maximal length of common words among random letter sequences. - Ann. Probab., 1988, v. 16, № 3, p. 535-563.

6. Arratia R., Waterman M.S. Critical phenomena in sequence matching. - Ann. Probab., 1985, v. 13, № 4, p. 1236-1249.

7. Arratia R., Waterman M.S. The Erdos-Renyi strong law for pattern matching with a given proportion of mismatches. - Ann. Probab., 1989, v. 17, № 3, p. 1152-1169.

8. Arratia R., Gordon L., Waterman M.S. The Erdos-Renyi law in distribution, for coin tossing and sequence matching. - Ann. Statist., 1990, v. 18, № 2, p. 539-570.

9. Roos M. Stein's method for compound Poisson approximation: The local approach. Ann. Appl. Probab., 1994, v. 4, № 4, p. 1177-1187.

10. Михайлов В.Г. Оценки точности сложной пуассоновской аппроксимации по методу Стейна-Чена. - Обозрение прикл. промышл. матем., сер. дискретн. матем., 1994 , т. 3 , в. 4 , с. $530-548$.

11. Barbour A.D., Chen L. H. Y., Loh W.-L. Compound Poisson approximation for nonnegative random variables via Stein's method. - Ann. Probab., 1992, v. 20, № 4, p. $1843-1866$.

12. Зубков А.М., Михайлов В.Г. Предельные распределения случайных величин, связанньх с длинными повторениями в последовательности независимьх испытаний. - Теория вероятн. и ее примен., 1974, т. 19, в. 1, с. 173-181.

13. Зубков A. М., Михайлов В.Г. О повторениях $s$-цепочек в последовательности независимых величин. - Теория вероятн. и ее примен., 1979, т. 24, в. 2, с. 267-279.

14. Barbour A. D., Holst L., Janson S. Poisson Approximation. Oxford: Clarendon Press, 1992, 277 p.

15. Smith R. L. Extreme value theory for dependent sequences via the Stein-Chen method of Poisson approximation. - Stochastic Process. Appl., 1988, v. 30, № 2, p. 317-327.

Поступила в редакцию 29.XII.1998

Исправленный вариант 5.VII.1999 